\title{
A Rare Presentation of Acute Alcohol Intoxication, Subarachnoid Haemorrhage and Cortical Venous Sinus Thrombosis
}

\author{
Harpreet Singh $^{\mathrm{a}}$, Paulomi Talapatra ${ }^{\mathrm{a}}$, Sucharita Ray ${ }^{\mathrm{a}, \mathrm{b}}$, Vikas Gupta ${ }^{\mathrm{a}}$
}

\begin{abstract}
We report a case of cortical venous sinus thrombosis presenting as subarachnoid haemorrhage in a young male with no underlying predisposition after a bout of acute alcohol intoxication. Radiological imaging proved him to have both subarachnoid hemorrhage and cortical venous thrombosis. After ruling out all differentials, a role of alcohol responsible for the dual occurrence of a cerebral cortical venous thrombosis with subarachnoid hemorrhage was thought and institution of therapy with low molecular weight heparin promptly improved his symptoms and with time he recovered and was discharged on warfarin. Warfarin was discontinued after 3 months and patient remains asymptomatic after 1 year of regular follow-up.
\end{abstract}

Keywords: Cortical venous thrombosis; Alcohol; Subarachnoid haemorrhage

\section{Introduction}

An elusive diagnosis, cerebral venous thrombosis (CVT) is increasingly recognised as a distinctive cause of cerebrovascular accident in the young. It results from occlusion of a

Manuscript accepted for publication April 6, 2012

${ }^{a}$ Department of Medicine, Pt. B. D. Sharma, PGIMS, Rohtak, India

${ }^{b}$ Corresponding author: 881/23, DLF Colony, Rohtak, Haryana-124001, India.Email: dr.sucharitaray@gmail.com

doi:10.4021/jnr97w venous sinus/or cortical vein, that results in cortical venous infarction with petechial or overt haemorrhagic perivascular venous infarctions. The clinical picture is extremely variable and presentations are often misleading. Isolated subarachnoid haemorrhage (SAH) and subdural hematoma (SDH) can be an uncommon presentation of CVT. Advent of newer imaging modalities have made the diagnosis easier in patients with high index of suspicion. Females are more commonly affected with a female to male ratio of 1.29:1. Common causes include prothrombotic states, infections, drugs and inflammatory diseases like Systemic Lupus Erythematosus, Behcet's disease etc. Alcoholism is an important risk factor commonly observed in male CVT patients [1]. We present a case of CVT in a chronic alcoholic that presented with subarachnoid haemorrhage.

\section{Case Report}

A 35 year old man presented to the emergency department with a one day history of headache and two episodes of complex partial seizures. He was addicted to alcohol for the past several years and had consumed alcohol continuously for the seven days before seizures began.

On examination, the patient was hemodynamically stable, responding to verbal commands, with no motor or sensory deficits and normally reacting pupils. Intermittent fine tremors were present on his hands with no evidence of ophthalmoplegia. In view of the above findings, a provisional diagnosis of alcohol withdrawal syndrome was made and treatment initiated accordingly.

Routine blood investigations including renal and liver function tests, serum electrolytes, prothrombin time, bleeding and clotting times were normal. Serum homocysteine, protein $\mathrm{C}$ and $\mathrm{S}$, antithrombin III, factor V Leiden and APLA were normal.

Despite intensive management, the patient's general condition dipped progressively until his GCS was $5 / 15$. A lumbar puncture revealed a csf that was uniformly bloodstained with fresh RBCs, sugar $50 \mathrm{mg} / \mathrm{dL}$ and protein 85 $\mathrm{mg} / \mathrm{dL}$. An urgent MRI brain was planned which revealed a thrombus in the superior sagittal sinus and bridging cerebral 


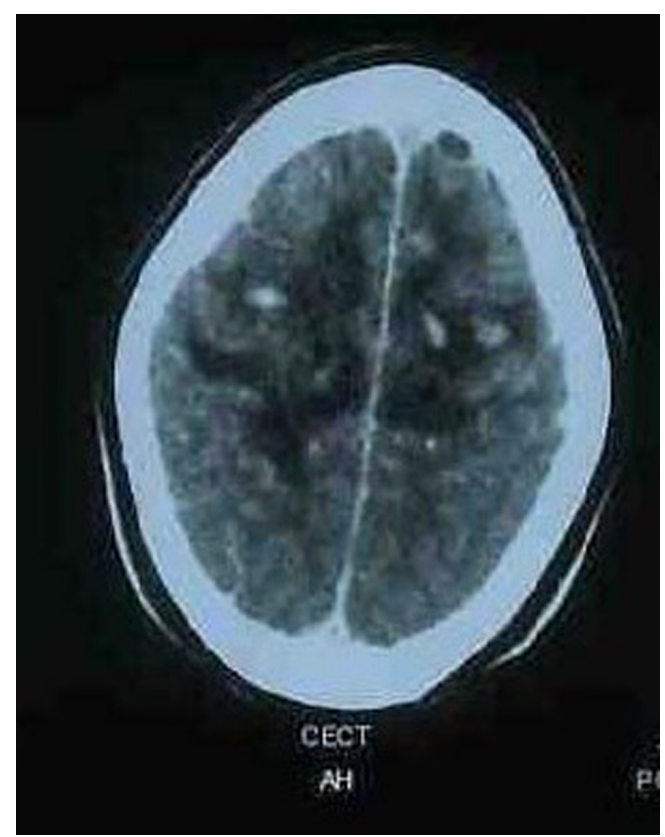

Figure 1. MRI brain showing a thrombus in the superior sagittal sinus and bridging cerebral veins with hemorrhagic infarcts in bilateral frontoparietal areas with blood in right sided sulci.

veins with hemorrhagic infarcts in bilateral frontoparietal areas with blood in right sided sulci suggestive of subarachnoid hemorrhage( Fig. 1). As a result, low molecular weight heparin was initiated along with the pre-existing antiedema measures, antiepileptics. His sensorium improved significantly after two days of treatment. Oral anticoagulation was initiated.

\section{Discussion}

The diagnosis of cerebral venous thrombosis (CVT) requires high index of suspicion owing to the varied spectrum of its clinical manifestations. Its diagnosis may be more confusing if the patient presents with subarachnoid hemorrhage (SAH). Risk factors include prothrombotic states, infection, drugs, inflammatory disease etc. Secondary hypercoaguable states are generally acquired disorders in patients with underlying systemic diseases or clinical conditions known to be associated with an increased risk of thrombosis such as malignancy, pregnancy, use of oral contraceptives, myeloproliferative disorders, hyperlipidemia, diabetes mellitus and abnormality of blood vessels [2].

Alcohol abuse has not been consistently found to be a risk factor for ischaemic stroke. Some studies have suggested a $\mathbf{J}$ shaped dose response curve between alcohol intake and a risk for stroke. In adults, chronic heavy drinking and acute alcohol intoxication have been associated with cerebral infarction, while moderate alcohol consumption seems to have a protective effect. Heavy drinking might lead to stroke by inducing hypertension, cardiac arrhythmias and hypercoagulability. It has also been seen that alcohol intake predisposes males for CVT by contributing to a state of dehydration and hyperviscosity and increasing platelet reactivity even if beverages like wine have been seen to exert a protective effect on the occurrence of ischaemic stroke when used in low doses [3]. Light drinking had no effect on the risk of SAH but decreased slightly the risk of ICH and CI. Heavy drinking increased the risk of all subtypes of stroke. Blood pressure and HDL-C seemed to be involved in the development of stroke. They did not, however, thoroughly explain the association between alcohol consumption and the risk of stroke; thus, the mechanisms of alcohol in stroke are basically still unexplained [4]. However the consensus statement in a metaanalysis on 35 observational studies indicate that heavy alcohol consumption increases the relative risk of stroke while light or moderate alcohol consumption may be protective against total and ischemic stroke [5].

CVT as a cause of SAH has been rarely reported in literature. Patients with CVT commonly present with severe headache and focal neurological symptoms and signs. CVT most commonly involves the superior sagittal sinus (72\%), followed by lateral sinus (70\%). In $30-40 \%$ cases, more than one sinus is involved with or without cortical venous thrombosis [5]. Imaging usually reveals single or multifocal parenchymal venous infarcts with haemorrhagic transformation and direct signs of CVT like dense delta and cord sign on plain CT, and empty delta sign on contrast enhanced CT [6].

SAH due to CVT is a low pressure, localised, cortical surface bleed which lacks the characteristics of a basal subarachnoid hemorrhage. Typical signs of basal SAH, such as nuchal rigidity, thunderclap headache or alteration of sensorium are rare. The multicompartmental intracranial bleed most probably results from rupture of bridging veins, secondary to high back pressure arising from the blockage of venous drainage into the thrombosed venous sinuses. Dural venous thrombosis with secondary venous hypertension may lead to SAH due to rupture of thin walled fragile cortical veins. Some of these veins may be dilated secondary to sinus thrombosis. Since, the SAH is due to the increased venous pressure following CVT, the site of rupture is perhaps in the cortical veins over the cerebral surface. This would explain the tendency for the subarachnoid blood to be seen in the brain convexities and the interhemispheric fissure [6]. Hence, an important radiological clue could be the sparing of the basal cisterns in SAH associated with CVT. CVT is a potentially serious, but fully treatable condition and its outcome depends upon early diagnosis and timely treatment. Recognition of the clinical and radiological signs can prevent delayed diagnosis of CVT even in the presence of apparent confounding presentations. It is important that such patients should also be enquired and investigated for risk factors for CVT like physical inactivity, alcohol abuse, 
puerperal state, infections of ear or sinuses, connective tissue disorders, hyperhomocysteinemia, prothrombotic states, drug abuse and hormone replacement therapy. Work-up for underlying primary thrombophilic state is ideally postponed to 8 - 12 weeks after the acute episode as these tests may be falsely negative or positive in the acute state, and the results are also affected by the administration of anticoagulant. Anticoagulants must be withdrawn at least for 2 weeks prior to testing for these abnormalities [7]. The patient improved with anticoagulant therapy despite subarachnoid bleed being present radiologically. Therefore in the exceptional circumstance of SAH in CVT, anticoagulation remains the mainstay of treatment [8].

\section{References}

1. Mathew T, Sarma GR, Kamath V, Roy AK. Subdural hematoma, subarachnoid hemorrhage and intracerebral parenchymal hemorrhage secondary to cerebral sinovenous thrombosis: a rare combination. Neurol India. 2007;55(4):438-439.

2. Moulakakis KG, Sfyroeras G, Pavlidis P, Besias N, Maras D, Andrikopoulos V. Hypercoagulable state due to alcohol-paracetamol syndrome producing acute limb ischemia. Vasc Endovascular Surg. 2007;41(4):362-365.

3. Malarcher AM, Giles WH, Croft JB, Wozniak MA, Wityk RJ, Stolley PD, Stern BJ, et al. Alcohol intake, type of beverage, and the risk of cerebral infarction in young women. Stroke. 2001;32(1):77-83.

4. Leppala JM, Paunio M, Virtamo J, Fogelholm R, Albanes D, Taylor PR, Heinonen OP. Alcohol consumption and stroke incidence in male smokers. Circulation. 1999;100(11):1209-1214.

5. Reynolds K, Lewis B, Nolen JD, Kinney GL, Sathya B, He J. Alcohol consumption and risk of stroke: a metaanalysis. JAMA. 2003;289(5):579-588.

6. Modi M, Singla V, Ahluwalia J, Sharma A, Prabhakar S, Khandelwal N, Duberkar D. HIV infection presenting as cerebral venous sinus thrombosis. Am J Emerg Med. 2012;30(3):517 e511-513.

7. Brousser MG, Barnett HJM. Cerebral Venous Thrombosis. In: Stroke: pathophysiology, diagnosis and management, 2nd ed. New York. Churchill Livingstone 1992; 517-37.

8. de Bruijn SF, Stam J, Kappelle LJ. Thunderclap headache as first symptom of cerebral venous sinus thrombosis. CVST Study Group. Lancet. 1996;348(9042):1623-1625. 